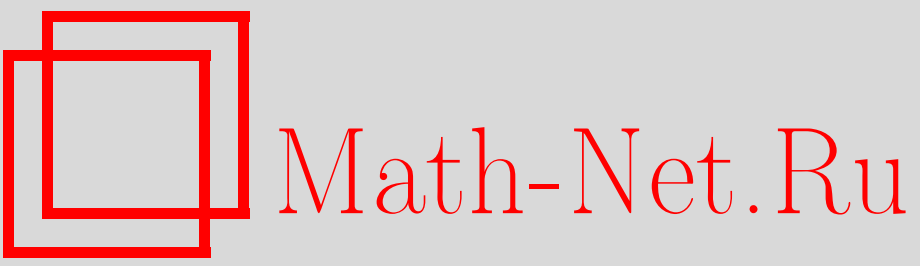

Е. А. Оноприенко, Об одном классе три-тканей Бола, Итоги науки и техн. Сер. Соврем. мат. и ее прил. Темат. обз., 2020, том 179, 37-40

DOI: https://doi.org/10.36535/0233-6723-2020-179-37-40

Использование Общероссийского математического портала Math-Net.Ru подразумевает, что вы прочитали и согласны с пользовательским соглашением

http://www.mathnet.ru/rus/agreement

Параметры загрузки:

IP: 54.162.27.143

26 апреля 2023 г., 12:26:24 


\title{
ОБ ОДНОМ КЛАССЕ ТРИ-ТКАНЕЙ БОЛА
}

\author{
(c) 2020 г. $\quad$ E. А. ОНОПРИЕНКО
}

\begin{abstract}
АннотАция. Рассматриваются инфинитезимальные свойства многомерных средних три-тканей Бола с ковариантно постоянным тензором кривизны (ткани $B_{m}^{\nabla}$ ); заложены основы классификации таких тканей по рангу тензора кручения. Для три-ткани $B_{m}^{\nabla}$ ранга $\rho$ методом Картана построен адаптированный репер и найдена соответствующая система структурных (дифференциальных) уравнений. Доказано, что три-ткань $B_{m}^{\nabla}$ ранга $\rho$ несет нормальную подткань, которая является групповой, причем соответствующая фактор-ткань является регулярной три-тканью. Путем интегрирования структурных уравнений найдены новые семейства примеров многомерных три-тканей специального типа и гладких луп Бола, являющихся обобщением полупрямого произведения двух абелевых групп Ли.
\end{abstract}

Ключевые слова: многомерная три-ткань, три-ткань Бола, групповая три-ткань, эластичная три-ткань, $G$-ткань, гладкая лупа Бола.

\section{ON ONE CLASS OF BOL THREE-WEBS}

\section{(c) 2020 E. A. ONOPRIENKO}

\begin{abstract}
In this paper, we consider infinitesimal properties of multidimensional mean Bol threewebs with a covariantly constant curvature tensor (webs $B_{m}^{\nabla}$ ) and lay the foundations for classifying such webs by the rank of the torsion tensor. For three-webs $B_{m}^{\nabla}$ of rank $\rho$, we construct an adapted frame by the Cartan method and find the corresponding system of structural (differential) equations. We prove that a three-web $B_{m}^{\nabla}$ of rank $\rho$ carries a normal subweb, which is a group web, and the corresponding factor-web is a regular three-web. By integrating the structure equations, we find new families of examples of multidimensional three-webs of a special type and smooth Bol loops, which are a generalization of the semidirect product of two Abelian Lie groups.
\end{abstract}

Keywords and phrases: multidimensional three-web, Bol three-web, group three-web, elastic threeweb, $G$-web, smooth Bol loop.

AMS Subject Classification: 53A60

В настоящей работе рассматриваются три-ткани Бола с ковариантно постоянным тензором кривизны.

Три-тканью называется совокупность трех попарно трансверсальных гладких слоений коразмерности $r$ на гладком многообразии $M$ размерности $2 r$. Локально слоения всегда можно задать уравнениями $x^{i}=$ const, $y^{i}=$ const и $f^{i}\left(x^{j}, y^{k}\right)=$ const; здесь и далее $i, j, k, \ldots=1,2, \ldots, r$. Локально три-ткань вполне определяется уравнением $z=f(x, y)$, которое связывает параметры $x, y, z$ слоев первого, второго и третьего слоений, проходящих через одну точку, и называется уравнением три-ткани. В силу трансверсальности слоев уравнение три-ткани разрешимо (локально) относительно переменных $x$ и $y$, поэтому оно определяет гладкую локальную квазигруппу, которая называется координатной квазигруппой три-ткани $W$. Следуя В. Бляшке, мы рассматриваем три-ткани с точностью до локальных диффеоморфизмов.

Систематическое изучение многомерных три-тканей начато в работе Черна [2]. Используя подход Картана-Лаптева, М. А. Акивис создал эффективный метод для изучения многомерных 
три-тканей. Этим методом получены все существенные результаты по теории многомерных тритканей (см. [1]).

Средние ткани Бола (ткани $B_{m}$ ) занимают особое место в теории тканей. Их алгебраический аналог - многомерные гладкие лупы Бола-следующие после аналитических луп Муфанг по близости своих свойств к группам Ли. Три-ткани $B_{m}$ обладают следующими свойствами:

1. они обладают замкнутой $G$-структурой класса 3 - ковариантные производные тензора кривизны являются комитантами тензоров кручения и кривизны;

2. на третьем слоении этих тканей естественным образом возникает структура симметрического пространства. Эта симметрия обладает специальными свойствами, которые изучались многими авторами.

Три-ткани $B_{m}$ были введены Г. Болом (см. [3]). Их алгебраические свойства исследовались В. Д. Белоусовым и другими авторами. Четырехмерные ткани Бола полностью описал А. Д. Иванов. Ткани $B_{m}$ произвольной размерности исследовала В. И. Федорова, которая, в частности, заложила основы классификации шестимерных тканей $B_{m}$. Специальный класс тканей Болаэластичные ткани, или ткани $E$ - исследовались А. М. Шелеховым, Г. Баландиной, К. Р. Джукашевым. Три-ткани $B_{m}$ с тензором кривизны минимального ранга рассматривались М. И. Антиповой. Гладкие лупы Бола исследовались Л. В. Сабининым и П. О. Михеевым.

Доказаны следующие утверждения.

1. Пусть кобазис дифференциальных форм $\underset{1}{\omega^{i}}$ и $\underset{2}{\omega^{i}}(i, j, k, \ldots=1, \ldots, r)$ на многообразии $M$ выбран так, что формы $\underset{1}{\omega^{i}}, \underset{2}{\omega^{i}}$ и $\underset{1}{\omega^{i}}+\underset{2}{\omega^{i}}$ аннулируются соответственно на первом, втором и третьем слоениях ткани $B_{m}^{\nabla}$. Тогда эти формы удовлетворяют системе дифференциальных уравнений

$$
\begin{aligned}
& d \omega_{1}^{i}=\underset{1}{\omega^{j}} \wedge \omega_{j}^{i}+a_{j k}^{i} \omega_{1}^{j} \wedge \underset{1}{\omega^{k},} \quad d \underset{2}{\omega^{i}}=\underset{2}{\omega_{j}^{j}} \wedge \omega_{j}^{i}-a_{j k}^{i}{\underset{2}{j}}_{j} \wedge \underset{2}{\omega^{k}} \\
& d \omega_{j}^{i}=\omega_{j}^{k} \wedge \omega_{k}^{i}+b_{j k l}^{i} \omega_{1}^{k} \wedge \underset{2}{\omega^{l}}, \quad \nabla a_{j k}^{i}=-b_{[j k] \ell}^{i}\left(\begin{array}{c}
\left.\omega_{1}^{\ell}-\omega_{2}^{\ell}\right) \\
\omega^{\ell}
\end{array}\right) \quad \nabla b_{j k \ell}^{i}=0,
\end{aligned}
$$

замкнутой относительно операции внешнего дифференцирования в силу соотношений

$$
\begin{gathered}
b_{[j k \ell]}^{i}=2 a_{[j k}^{m} a_{|m| \ell]}^{i}, \quad b_{j(k l)}^{i}=0, \quad b_{j k p}^{i} a_{\ell m}^{p}=0, \\
a_{j k}^{p} b_{p \ell m}^{i}=a_{p k}^{i} b_{j \ell m}^{p}+a_{j p}^{i} b_{k \ell m}^{p}, \quad b_{j k p}^{i} b_{l m q}^{p}=0, \quad b_{p r s}^{i} b_{j k l}^{p}=b_{p k l}^{i} b_{j r s}^{p} .
\end{gathered}
$$

Здесь $a_{j k}^{i}$ - тензор кручения, $b_{j k l}^{i}$-тензор кривизны.

2. Не существует негрупповых четырехмерных тканей $B_{m}^{\nabla}$.

3. Класс негрупповых шестимерных тканей $B_{m}^{\nabla}$ содержит только две три-ткани - это известные эластичные ткани $E_{1}$ и $E_{2}$.

Пусть $\mathcal{A}$ - алгебра, определяемая тензором кручения. Назовем рангом тензора кручения размерность производной алгебры $\mathcal{A}^{\prime}$. Верно следующее утверждение:

4. Ткани $B_{m}^{\nabla}$ с тензором кручения ранга $0, r$ или $r-1$ являются групповыми тканями.

Анализ системы (1)-(2) приводит к следующей теореме.

5. Класс тканей $B_{m}^{\nabla}$ с тензором кручения ранга 1 совпадает с классом эластичных тканей $E_{1}^{r}$.

Уравнения ткани $E_{1}^{r}$ в локальных координатах имеют вид

$$
z^{1}=x^{1}+e^{2 a_{\hat{k}} x^{\hat{k}}}\left(y^{1}+\lambda_{\hat{j} \hat{k}} x^{\hat{j}} y^{\hat{k}}\right), z^{\hat{i}}=x^{\hat{i}}+y^{\hat{i}},
$$

где $\hat{i}, \hat{j}, \hat{k}=2, \ldots, r, a_{\hat{k}}$ и $\lambda_{\hat{j} \hat{k}}-$ постоянные, причем $\lambda_{\hat{j} \hat{k}}=-\lambda_{\hat{k} \hat{j}}$. 
6. Для ткани $B_{m}^{\nabla}$ ранга $\rho$ существует адаптированный репер, в котором структурные уравнения этой ткани имеют вид

$$
\begin{aligned}
& d \omega_{1}^{a}=\omega_{1}^{b} \wedge \omega_{b}^{a}+\omega_{1}^{v} \wedge \omega_{v}^{a}+a_{j k}^{a} \omega_{1}^{j} \wedge{\underset{1}{\omega}}^{k}, \\
& d \omega_{2}^{a}=\omega_{2}^{b} \wedge \omega_{b}^{a}+\omega_{2}^{v} \wedge \omega_{v}^{a}-a_{j k}^{a} \omega_{2}^{j} \wedge{\underset{2}{\omega}}^{k}, \\
& d \omega^{u}=0 \\
& d \omega_{2}^{u}=0 \\
& d \omega_{b}^{a}=\omega_{b}^{c} \wedge \omega_{c}^{a}+2\left(a_{w z}^{c} a_{c b}^{a}+a_{z b}^{c} a_{c w}^{a}+a_{b w}^{c} a_{c z}^{a}\right) \omega_{1}^{w} \wedge \underset{2}{\omega^{z},} \\
& d \omega_{u}^{a}=\omega_{u}^{c} \wedge \omega_{c}^{a}+b_{u w z}^{a} \omega_{1}^{w} \wedge \omega_{2}^{z},
\end{aligned}
$$

причем ненулевые компоненты тензоров кручения и кривизны связаны соотношениями

$$
\begin{aligned}
& a_{d e}^{b} a_{b c}^{a}+a_{e c}^{b} a_{b d}^{a}+a_{c d}^{b} a_{b e}^{a}=0, \quad a_{d u}^{b} a_{b c}^{a}+a_{u c}^{b} a_{b d}^{a}+a_{c d}^{b} a_{b u}^{a}=0 ; \\
& a_{b u}^{f}\left(a_{f d}^{a} a_{v c}^{b}+a_{c f}^{a} a_{v d}^{b}-a_{c d}^{b} a_{f v}^{a}\right)+a_{b v}^{f}\left(a_{f d}^{a} a_{c u}^{b}+a_{c f}^{a} a_{d u}^{b}-a_{c d}^{b} a_{f u}^{a}\right)=0 ; \\
& \left(a_{u v}^{d} a_{d b}^{a}+a_{v b}^{d} a_{d u}^{a}+a_{b u}^{d} a_{d v}^{a}\right)\left(a_{w z}^{e} a_{e c}^{b}+a_{z c}^{e} a_{e w}^{b}+a_{c w}^{e} a_{e z}^{b}\right)= \\
& =\left(a_{w z}^{d} a_{d b}^{a}+a_{z b}^{d} a_{d w}^{a}+a_{b w}^{d} a_{d z}^{a}\right)\left(a_{u v}^{e} a_{e c}^{b}+a_{v c}^{e} a_{e u}^{b}+a_{c u}^{e} a_{e v}^{b}\right) ; \\
& b_{b u v}^{a}=2\left(a_{u v}^{c} a_{c b}^{a}+a_{v b}^{c} a_{c u}^{a}+a_{b u}^{c} a_{c v}^{a}\right) ; \\
& b_{u v w}^{a}+b_{v w u}^{a}+b_{w u v}^{a}=2\left(a_{u v}^{b} a_{b w}^{a}+a_{v w}^{b} a_{b u}^{a}+a_{w u}^{b} a_{b v}^{a}\right) ; \\
& a_{c b}^{a} b_{w u v}^{b}=2 a_{c w}^{b}\left(a_{u v}^{f} a_{f b}^{a}+a_{v b}^{f} a_{f u}^{a}+a_{b u}^{f} a_{f v}^{a}\right)-2 a_{b w}^{a}\left(a_{u v}^{f} a_{f c}^{b}+a_{v c}^{f} a_{f u}^{b}+a_{c u}^{f} a_{f v}^{b}\right) ; \\
& a_{b z}^{a} b_{w u v}^{b}+a_{w b}^{a} b_{z u v}^{b}=2 a_{w z}^{b}\left(a_{u v}^{c} a_{c b}^{a}+a_{v b}^{c} a_{c u}^{a}+a_{b u}^{c} a_{c v}^{a}\right) ; \\
& b_{q w z}^{b}\left(a_{v b}^{c} a_{c u}^{a}+a_{b u}^{c} a_{c v}^{a}\right)-b_{q u v}^{b}\left(a_{z b}^{c} a_{c w}^{a}+a_{b w}^{c} a_{c z}^{a}\right)= \\
& =2 a_{w z}^{c}\left[a_{c q}^{b}\left(a_{u v}^{f} a_{f b}^{a}+a_{v b}^{f} a_{f u}^{a}+a_{b u}^{f} a_{f v}^{a}\right)-a_{b q}^{a}\left(a_{u v}^{f} a_{f c}^{b}+a_{v c}^{f} a_{f u}^{b}+a_{c u}^{f} a_{f v}^{b}\right)\right]- \\
& -2 a_{u v}^{c}\left[a_{c q}^{b}\left(a_{w z}^{f} a_{f b}^{a}+a_{z b}^{f} a_{f w}^{a}+a_{b w}^{f} a_{f z}^{a}\right)-a_{b q}^{a}\left(a_{w z}^{f} a_{f c}^{b}+a_{z c}^{f} a_{f w}^{b}+a_{c w}^{f} a_{f z}^{b}\right)\right]
\end{aligned}
$$

и удовлетворяют дифференциальным уравнениям

$$
\begin{aligned}
& \nabla a_{b c}^{a}=d a_{b c}^{a}+a_{b c}^{d} \omega_{d}^{a}-a_{d c}^{a} \omega_{b}^{d}-a_{b d}^{a} \omega_{c}^{d}=0 ; \\
& \nabla a_{b u}^{a}=d a_{b u}^{a}+a_{b u}^{d} \omega_{d}^{a}-a_{d u}^{a} \omega_{b}^{d}-a_{b d}^{a} \omega_{u}^{d}=-\left(a_{u v}^{c} a_{c b}^{a}+a_{v b}^{c} a_{c u}^{a}+a_{b u}^{c} a_{c v}^{a}\right)\left(\underset{1}{\omega^{v}}-{\underset{2}{\omega}}^{v}\right) ; \\
& \nabla a_{u v}^{a}=d a_{u v}^{a}+a_{u v}^{d} \omega_{d}^{a}-a_{d v}^{a} \omega_{u}^{d}-a_{u d}^{a} \omega_{v}^{d}=-b_{[u v] w}^{a}\left(\begin{array}{c}
\omega_{1}^{w}-\omega_{2}^{w} \\
1
\end{array}\right) \\
& \nabla a_{u v}^{a}=d a_{u v}^{a}+a_{u v}^{d} \omega_{d}^{a}-a_{d v}^{a} \omega_{u}^{d}-a_{u d}^{a} \omega_{v}^{d}=\left(\frac{1}{2} b_{w u v}^{a}-\left(a_{u v}^{b} a_{b w}^{a}+a_{v w}^{b} a_{b u}^{a}+a_{w u}^{b} a_{b v}^{a}\right)\right)\left(\underset{1}{\omega^{w}}-\omega_{2}^{w}\right) ; \\
& \nabla b_{c u v}^{a}=d b_{c u v}^{a}+b_{c u v}^{b} \omega_{b}^{a}-b_{b u v}^{a} \omega_{c}^{b}=0 \\
& \nabla b_{w u v}^{a}=d b_{w u v}^{a}+b_{w u v}^{b} \omega_{b}^{a}-b_{b u v}^{a} \omega_{w}^{b}=0 .
\end{aligned}
$$

Эта система замкнута относительно внешнего дифференцирования.

7. В построенном адаптированном репере тензор кручения ткани $B_{m}^{\nabla}$ удовлетворяет, помимо обобщенного тождества Якоби, еще некоторым соотношениям третьей и четвертой степени.

8. Три-ткань $B_{m}^{\nabla}$ ранга $\rho$ допускает нормальную подткань $\widetilde{W}$, которая является групповой, причем соответствующая фактор-ткань $W_{1}=W / \widetilde{W}$ является регулярной.

$\mathrm{B}$ настоящей работе исследуются ткани $C B_{m}^{\nabla}$, для которых $a_{b c}^{a}=0$. В этом случае справедливо следующее утверждение.

9. Операторы $A_{u}=\left(a_{b u}^{a}\right)$ порождают линейную алгебру Ли $[\overline{\mathcal{A}}]$. Эта алгебра является нильпотентной высоты 2. 
Полностью описаны ткани, у которых алгебра $[\overline{\mathcal{A}}]$ является тривиальной, то есть все операторы $A_{u}$ попарно коммутируют. Эти ткани обозначаем $C B_{m}^{\nabla}(1)$ - общий случай, и $C B_{m}^{\nabla}(0)-$ все операторы $A_{u}$ имеют простую структуру.

10. Для тканей $C B_{m}^{\nabla}(0)$ существует семейство адаптированных реперов, в которых все операторы $A_{u}$ становятся скалярными. Три-ткань $C B_{m}^{\nabla}(0)$ обладает замкнутой $G_{W}$-структурой класса 2 и является $G$-тканью, то есть допускает транзитивную группу автоморфизмов. При некотором выборе параметров слоений уравнения три-ткани $C B_{m}^{\nabla}(0)$ приводятся к виду

$$
\begin{aligned}
& Z^{a}=X^{a}+e^{2 a_{a u}^{a} X^{u}}\left(Y^{a}-\frac{1}{4} \lambda_{v z}^{a}\left(X^{v} Y^{z}-X^{z} Y^{v}\right)\right), \\
& Z^{u}=X^{u}+Y^{u} .
\end{aligned}
$$

Найдена система структурных уравнений тканей $C B_{m}^{\nabla}(1)$ и проинтегрирована.

11. В некоторых локальных координатах уравнения ткани $C B_{m}^{\nabla}(1)$ имеют вид:

$$
\begin{aligned}
Z^{a}= & X^{a}+e_{b}^{a}(X)\left(Y^{b}-\frac{1}{2} d_{u v}^{b}\left(X^{u} Y^{v}-X^{v} Y^{u}\right)-\Lambda_{(u w) v}^{b} X^{u} X^{w} Y^{v}\right)+ \\
& +\frac{2}{3} S_{c}^{a}(X) a_{b(t}^{c} \Lambda_{u w) v}^{b} X^{t} X^{u} X^{w} Y^{v} \\
Z^{u}= & X^{u}+Y^{u} .
\end{aligned}
$$

Этот класс тканей зависит от параметров $a_{b u}^{a}$-компонент тензора кручения, входящих в функции $e_{b}^{a}(X)$ и $S_{c}^{a}(X)$, постоянных $d_{u v}^{b}$ и $\Lambda_{u v w}^{b}$. Функции $S_{c}^{a}(X)$ находятся из дифференциальных уравнений

$$
S^{\prime}(x) C(x)+3 S(x)=3 \exp (2 C(x)),
$$

где штрих обозначает формальную производную по $C, C(x)=\left(a_{b u}^{a} x^{u}\right)$, и удовлетворяют уравнению

$$
S(x) C^{3}(x)=\frac{3}{2} \exp (2 C(x))\left(C(x)-\frac{1}{2} E\right)^{2} .
$$

\section{СПИСОК ЛИТЕРАТУРЫ}

1. Akivis M. A., Shelekhov A. M. Geometry and Algebra of Multidimensional Three-Webs. - DordrechtBoston-London: Kluwer, 1992.

2. Chern S. S. Eine Invariantentheorie der Dreigewebe aus $r$-dimensionalen Mannigfaltigkeiten in $\mathbb{R}_{2 r} / /$ Abh. Math. Sem. - 1936. - 11, № 1-2. - P. 333-358.

3. Bol G. Gewebe und Gruppen// Math. Ann. - 1937. - 114. - P. 414-431.

Оноприенко Екатерина Андреевна

Бауманская инженерная школа № 1580, Москва

E-mail: katrinonoprienko@mail.ru 